\title{
Acta
Biochimica
Polonica
}

Vol. 47 No. 2/2000

$459-467$

QUARTERLY

\section{Reversion of argE3 ochre strain Escherichia coli AB1157 as a tool for studying the stationary-phase (adaptive) mutations}

\author{
Anetta Nowosielska and Elżbieta Grzesiuk ${ }^{\bowtie}$
}

Institute of Biochemistry and Biophysics, Polish Academy of Sciences, Warszawa, Poland

Received: 21 January, 2000; accepted: 27 March, 2000

Key words: adaptive mutations, $\mathrm{Arg}^{+}$revertants, Escherichia coli, mutT, mutY, replica plating

\begin{abstract}
Adaptive (starvation-associated) mutations occur in non-dividing cells and allow growth under the selective conditions imposed. We developed a new method for the determination of adaptive mutations in Escherichia coli. The system involves reversion to prototrophy of the $\operatorname{argE~}_{\mathrm{OC}}$ mutation and was tested on $\mathrm{AB1157}$ strains mutated in the $m u t T$ and/or $m u t Y$ genes. The bacteria that mutated adaptively grow into colonies on minimal medium plates devoid of arginine (starvation conditions) when incubated longer than 4 days. Using the replica plating method we solved the problem of discrimination between growth-dependent and adaptive $\operatorname{argE3} \rightarrow \mathrm{Arg}^{+}$revertants. Phenotype analysis and susceptibility of the $\mathrm{Arg}^{+}$revertants to a set of T4 phage mutants create an additional possibility to draw a distinction between these two types of $\mathrm{Arg}^{+}$revertants.
\end{abstract}

Adaptive mutations (also called "directed", "stationary-phase" or "starvation-associated") are a special kind of spontaneous mutations that occur in non-dividing or slowly-growing stationary-phase cells. Mutations of this type are detectable after exposure to a non-lethal selection and have been found only in genes whose functions were selected for (e.g. [1-5]). The last part of this definition is rather controversial especially as one of the most extensively used experimental systems for the investigation of adaptive mutations has recently been shown not to be strictly adaptive because, by using appropriate methods, mutations in non selected loci could also be found [6]. This system uses the reversion of the lacI33-1 frameshift mutation carried on the F' sex plasmid in the FC40 strain of Escherichia coli $[7,8]$. The lacI33 $\rightarrow \mathrm{Lac}^{+}$reversion, if plasmid borne, depends on rec $A B C$ genes [9]. When lacI33 mutation is localized on the chromosome it reverts adaptively at a much lower rate and the event is recA independent. This means that the system is a special case which

${ }^{\circledR}$ Corresponding author: Dr. Elżbieta Grzesiuk, Institute of Biochemistry and Biophysics, PAS, A. Pawińskiego 5A, 02-106 Warszawa, Poland; tel: (48 22) 658 4766; fax: (48 22) 3912 1623; e-mail: elag@ibbrain.ibb.waw.pl 
is related to plasmid metabolism rather than to chromosomal DNA.

The systems operating on bacterial chromosomal loci use reversion to prototrophy of auxotrophic E. coli strains. Prototrophic revertants are able to grow on minimal medium plates lacking the previously required components (e.g. [10]). Adaptive mutations have been reported, i.e., for $\operatorname{trp} A, \operatorname{trp} B$ [4], $\operatorname{trp} E[10]$ and tyrA $[11,12]$.

In this report we present a new system for adaptive mutation investigation in $E$. coli, the $\arg E 3_{\mathrm{OC}} \rightarrow \mathrm{Arg}^{+}$reversion to prototrophy, supplemented with the replica plating. This replica plating method described by Miller [13] was applied for a new purpose, namely, the discrimination between colonies which grew on selective plates as a result of creation of growth-dependent mutations, and those formed after prolonged starvation in the absence of regular DNA replication. An additional advantage of the system, verified on two E. coli mutants: mutT and mutY, is the simple way of estimation of the specificity of mutations.

\section{MATERIALS AND METHODS}

Bacterial strains. All bacteria were derivatives of $E$. coli K12 strain AB1157 (genotype: $\mathrm{F}^{-}$thr-1 leuB6 proA2 his4 thi1 argE3 lacY1 galK2 rpsL supE44 ara-14 xyl-15 mtl-1, txs-33 [14]). AB1157mutT was obtained by P1 transduction of leu::Tn10 mutT from JM105mutT; AB1157mutY by transduction of mutY68::miniTn10 from BH980. Bacterial donors of mutT and mutY were from Drs. S. Boiteux (Dept. de Radiobiologie et Radiopathologie, France) and J. Laval (Institut Gustave Roussy, France), respectively. The double mutant AB1157mutTmutY was constructed by A. Wójcik [15].

Mutation experiments. Bacteria were grown overnight at $37^{\circ} \mathrm{C}$ with shaking in $\mathrm{E}$ medium (E medium consisted of C-salts [16] sup- plemented with glucose $(0.5 \%)$, casamino acids $(0.2 \%)$, Thi $(10 \mu \mathrm{g} / \mathrm{ml})$ and arginine, histidine, threonine, proline and leucine at 25 $\mu \mathrm{g} / \mathrm{ml}$ each), centrifuged and resuspended in C-salts. Aliquots, at 3-4 $\times 10^{8}$ and 3-4 $\times 10^{7}$ cells/plate (the number of viable bacteria was estimated by dilution and plating on LB medium solidified with 1.5\% Difco Bacto agar) were plated onto E-Arg (E minus arginine) solid medium. Plates were sealed with Parafilm and incubated at $37^{\circ} \mathrm{C}$. $\mathrm{Arg}^{+}$colonies were counted first after $48 \mathrm{~h}$ and then their number was checked each day up to 10 days. The number of viable cells on starvation plates was determined as follows: the colonies which appeared on E-Arg plates were removed with a cork borer and the bacteria were washed off with $5 \mathrm{ml}$ of $\mathrm{E}$ medium, diluted and plated on LB-agar plates. $\mathrm{Arg}^{+}$revertants were analyzed as described before [17, 18]. Briefly, 100 colonies were tested for arginine, histidine and threonine requirements, and 50 colonies from class I, $\mathrm{Arg}^{+} \mathrm{His}^{-} \mathrm{Thr}^{-/+}$and class II, $\mathrm{Arg}^{+} \mathrm{His}^{+} \mathrm{Thr}^{-/+}$were tested for their susceptibility to a set of amber and ochre T4 phages (Table 1) to identify tRNA suppressors and deduce the specificity of mutations

Replica plating was a modification of the method described by Miller [13]. Overnight cultures of AB1157 strains were plated on E-Arg plates devoid of arginine (master plates) and incubated at $37^{\circ} \mathrm{C}$ for 2 days, the time necessary for growth-dependent $\mathrm{Arg}^{+}$ revertants to grow into colonies on minimal medium plates. After 2 days three or four $\mathrm{E}^{-}$Arg replica plates were prepared from each master plate (for replica plates a replicating block covered with velvet was used). These plates were incubated for 3 days. The distribution of the $\mathrm{Arg}^{+}$growth-dependent colonies was identical on the master and replica plates because a large number of non-starved, vigorously replicating cells were transferred from the colonies already existing on a master plate to replica plates. In addition to these colonies, single cells were transferred through the vel- 
vet to replica plates but each of the cells has mutated at different time under starvation conditions and, as a result, the bacteria which had mutated adaptively grew on each plate in different places.

\section{RESULTS AND DISCUSSION}

\section{A method for the discrimination between growth-dependent and adaptive $\mathrm{Arg}^{+}$ revertants}

We used the replica plate test to distinguish between growth-dependent and adaptive colonies. Figure 1 presents a picture of a master plate (after 2 and 5 days of incubation at $37^{\circ} \mathrm{C}$ ) and three replica plates. The master plate contained $10^{7}$ cells of $E$. coli strain AB1157mutT. On the plate photographed after two days of incubation only growth-dependent $\mathrm{Arg}^{+}$colonies can be seen, but after the next 3 days of incubation additional, small colonies appeared both on the master plate and on the replica plates. Large, growth-dependent colonies formed exactly the same pattern on all plates (the circles in Fig. 1 surround three growth-dependent colonies on every plate). Small colonies which appeared after the $5^{\text {th }}$ day of plate incubation were distributed in a different way on the master and replica plates (the circle in Fig. 1C shows three colonies which did not appear on the remaining plates). This can be explained as follows: during the slow process of DNA turnover individual cells randomly distributed on each plate mutate in the $\arg E$ gene which allows them to form $\mathrm{Arg}^{+}$colonies. Since the adaptive $\operatorname{argE3} \rightarrow \mathrm{Arg}^{+}$reversions arise independently on each replica plate the "adaptive" colonies are located on each plate in different places. This test seems to be especially useful in the case of slow growing growth-dependent colonies, which, unlike the adaptive ones, were pre-formed on the master plate and consequently create an identical pattern on all replica plates.

\section{Adaptive $\operatorname{argE} 3_{\mathrm{OC}} \rightarrow \mathrm{Arg}^{+}$reversion in $\mathrm{mutT}$ and mutY strains}

In this study we used $\arg E 3 \rightarrow \mathrm{Arg}^{+}$reversion to prototrophy as an indicator of the spontaneous mutagenesis rate under starvation conditions and two other reversions: his G4 $\rightarrow \mathrm{His}^{+}$ and $t h r^{-} \rightarrow \mathrm{Thr}^{+}$to estimate the adaptive mutation specificity.

To test the system on a higher number of revertants, additional mutations increasing the level of spontaneous reversions had to be introduced into the bacterial chromosome, because the level of spontaneous $\mathrm{Arg}^{+}$reversions in the AB1157 strain is very low (3-5 colonies $/ 10^{8}$ cells). In the system of reversion to prototrophy of tyrA14 [11], trpA23 [12] or trpE65 [19], mutations in mutT or mutY genes lead to an elevated mutation rate in starved bacteria. The product of the mutT gene, the MutT protein, hydrolyses 8-oxo-dGTP, generated by active oxygen species, to the nucleoside diphosphate, thus cleansing the triphosphate pool of this contaminant [20]. The lack of the MutT protein leads to a high rate of spontaneous mutations. The MutY protein is an adenine DNA-glycosylase that removes adenine from an A:8-oxoG pair. 8-OxoG is a DNA lesion resulting from attack by various oxidizing species and its presence leads to $\mathrm{GC} \rightarrow \mathrm{TA}$ transversions by directing the incorporation of adenine [21].

For the investigation of adaptive mutations, AB1157 bacteria from an overnight culture were plated on minimal medium plates lacking arginine (E-Arg). The $\mathrm{Arg}^{+}$colonies started to appear after 2 days of incubation at $37^{\circ} \mathrm{C}$. There were not more than $2-3$ colonies at the beginning of the experiment and 14-20 at the end of the 10-day period (Fig. 2). The presence of mutT or mutY mutations increased the number of growth-dependent as well as adaptive $\mathrm{Arg}^{+}$revertants.

Figure 3 shows the effect of the mutT mutation on the appearance of the $\operatorname{argE} 3_{\mathrm{OC}} \rightarrow \mathrm{Arg}^{+}$ revertants during 10 days of incubation under starvation conditions. AB1157mutT bacteria 

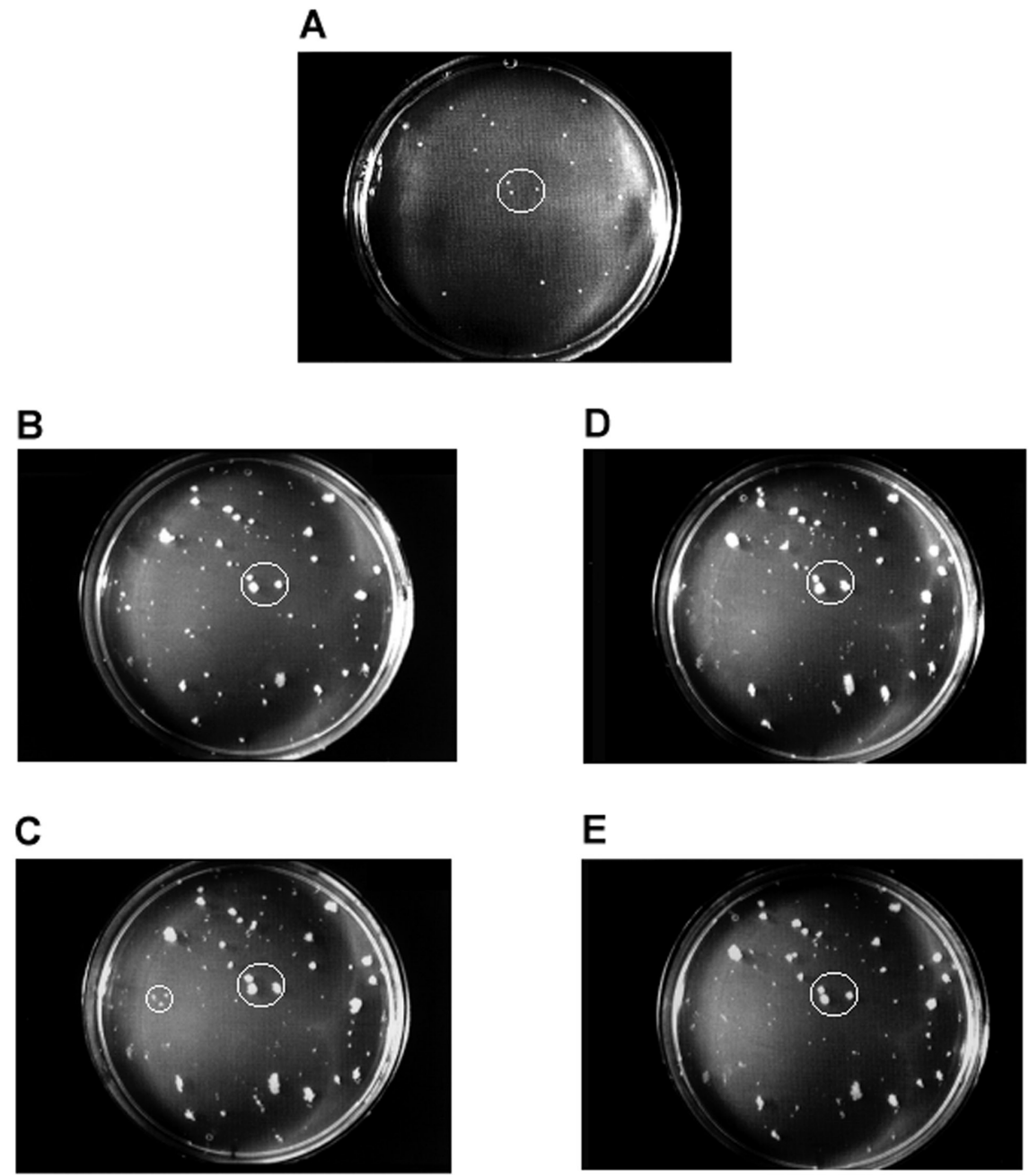

Figure 1. Replica plate test on AB1157mutT strain.

A, master plate after 2 days of incubation at $37^{\circ} \mathrm{C}$. B, master plate after 5 days of incubation at $37^{\circ} \mathrm{C}$. C, D, E, replica plates after 3 days of incubation at $37^{\circ} \mathrm{C}$. The 3 colonies surrounded by a circle on each plate are an example of Arg ${ }^{+}$ growth-dependent revertants. The 3 colonies in a smaller circle on plate $\mathrm{C}$ are an example of $\mathrm{Arg}^{+}$adaptive revertants.

were plated at two densities: $10^{8}$ and $10^{7}$ cells/plate. In resting cells under selection conditions $\mathrm{Arg}^{+}$revertants appeared at a rate between 100 and 190 colonies per day (average $153 \pm 35$ ) when $10^{8}$ bacteria were plated, and between 46 and 200 (average $94 \pm 17$ ) when the density of $10^{7}$ was used. The overall increase in the mutation rate was 3 - and 14-fold for $10^{8}$ and $10^{7}$ cells/plate, respectively.

The kinetics of adaptive mutation growth rate in the $\mathrm{AB} 1157$ mut $Y$ strain was different (Fig. 3). The density of $10^{7}$ bacteria/plate gave not more than 10 colonies at the end of the incubation period, but $10^{8}$ cells produced over 150 colonies/plate. The large number of starvation-associated mutations in the absence of MutY protein could originate from the presence of unrepaired bases such as 8-oxoG which occur frequently in starved cells. The results obtained here, in the $\arg E 3 \rightarrow \mathrm{Arg}^{+}$reversion to prototrophy system are similar to those reported for mut $T$ in tyrA14 and trpA23 [12] and for mutY in tyrA14 [11] systems.

Surprisingly, in the AB1157mutTmutY double mutant the level of adaptive mutations was low and comparable to that observed in 
wild type AB1157 (Fig. 2). As expected, the level of growth-dependent $\mathrm{Arg}^{+}$revertants in the mutTmut $Y$ strain was very high (about 770 \pm 15 mutants per $10^{8}$ cells).

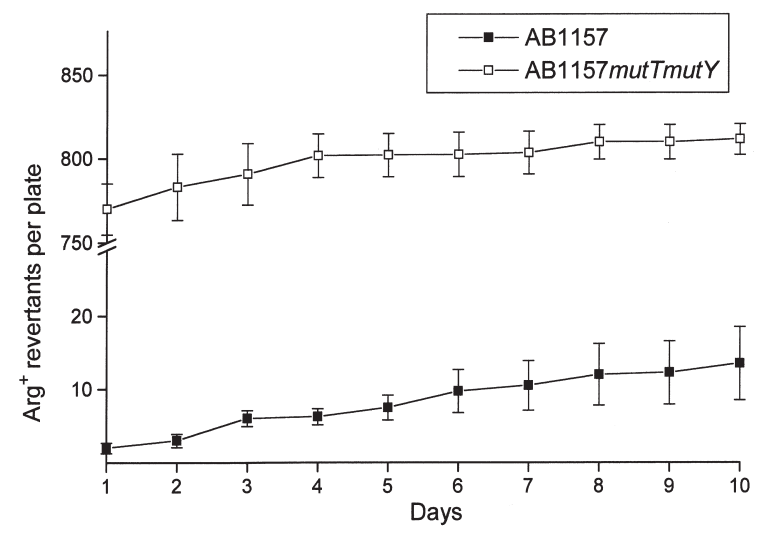

Figure 2. $\mathrm{Arg}^{+}$revertants of $\mathrm{AB} 1157$ and $\mathrm{AB} 1157$ mutTmut $Y$ strains in $10^{8}$ cells as a function of the duration of incubation at $37^{\circ} \mathrm{C}$ on E-Arg plates.

Points represent means, and bars - standard errors of three or four experiments.

In all the described experiments it was important to check whether the cells on starvation plates were alive. After removal of all visi-

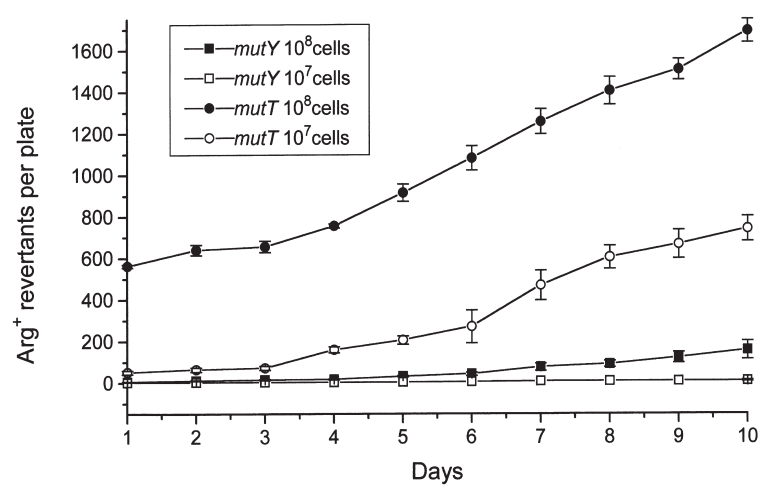

Figure 3. Accumulation of $\mathrm{Arg}^{+}$adaptive mutations over time (incubation at $37^{\circ} \mathrm{C}$ ) on E-Arg plates in AB1157 mutT and mutY strains at $10^{7}$ and $10^{8}$ cells/plate.

Points represent means, and bars - standard errors of three or four experiments.

ble colonies, the plates were washed and the number of viable cells was estimated by diluting the obtained suspensions and plating onto
LB-agar plates. Figure 4 shows the viability of AB1157 wild type strain and its mutY and mutT mutants on E-Arg plates $\left(10^{8}\right.$ cells were placed on each plate). The number of viable

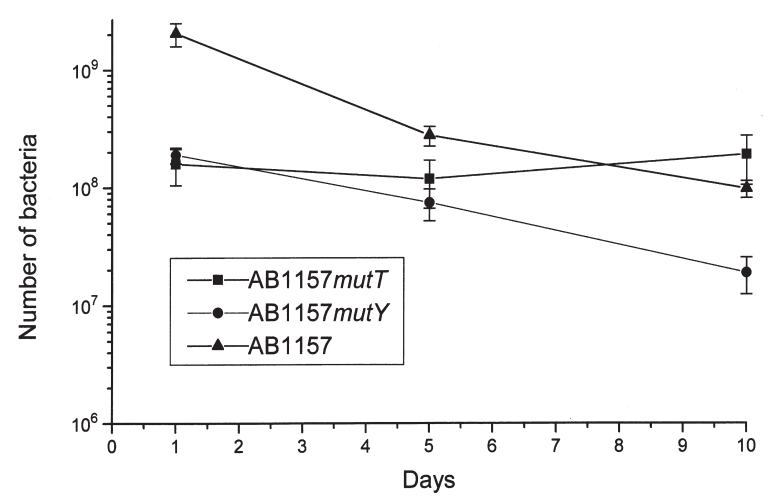

Figure 4. Viable counts of AB1157 wild type, mut $T$ and mutY strains.

Bacteria were washed off from of E-Arg plates after 1, 5 and 10 days of incubation at $37^{\circ} \mathrm{C}$.

cells was estimated at the beginning of the experiment, on the $1^{\text {st }}$ day and after 5 and 10 days of plate incubation. Viable counts of mut $Y$ and $m u t T$ strains were not significantly changed during the first 5 days of incubation. However, after 10 days the number of living mut $Y$ cells decreased to reach a 10 -fold lower number in comparison to the initial counts, while the number of viable mutT cells stayed more or less on the same level. Since the drop in the number of living cells in mutY mutant is very similar to that in wild type strain it can be concluded that the mutation in mutY gene has no influence on cell viability and that the mutation in mutT gene helps bacterial cells to survive under prolonged starvation conditions.

\section{Specificity of adaptive mutations}

Previously, we have described a method for estimating mutagenic specificity in $E$. coli AB1157 strain carrying three mutations: argE3 (ochre), hisG4 (ochre) and thr-1 (amber) [18]. The method is based upon analysis of the $\mathrm{Arg}^{+}$revertants which may arise by back mu- 
tations at the mutated site (UAA), or by ochre suppressor formation. One of the advantages of the method is that it allows investigation of the mutagenic processes on the intact chromosomes.

In the present experiments two sets of $\mathrm{Arg}^{+}$ colonies were tested for their specificity: the colonies which appeared after 2 days of incubation at $37^{\circ} \mathrm{C}$ (growth-dependent revertants), and those which appeared between the $4^{\text {th }}$ and $10^{\text {th }}$ day of incubation (stationary-phase mutations). First, the $\mathrm{Arg}^{+}$revertants selected on $\mathrm{E}$-Arg plates were examined for their requirements for histidine and threonine by plating them on E-His and E-Thr plates. All the Arg ${ }^{+}$ revertants can be divided into two phenotypic classes: class I: $\mathrm{Arg}^{+} \mathrm{His}^{-} \mathrm{Thr}^{+/-}$; class II, $\mathrm{Arg}^{+} \mathrm{His}^{+} \mathrm{Thr}^{+/-}$. The suppressor activity of the $\mathrm{Arg}^{+}$revertants can be examined by testing their sensitivity to a set of amber (B17) and ochre (oc427, ps292, ps205) mutants of the $\mathrm{T} 4$ bacteriophage. $\mathrm{T} 4$ mutants are able to grow only on the $\mathrm{Arg}^{+}$revertants creating a suppressor (the results of phage typing precisely point to the suppressor present, see Table 1). The $\mathrm{Arg}^{+}$revertants which are not sen-
AB1157 strain $\mathrm{Arg}^{+}$growth-dependent revertants were almost evenly distributed between phenotypic class I and II. Class I revertants were a result of $\sup B$ suppressor formation by $\mathrm{GC} \rightarrow \mathrm{AT}$ transitions while class II were due to supL suppressor formation by AT $\rightarrow$ TA transversions. Arg $^{+}$adaptive revertants of the same strain belonged mainly to class I and were a result of the formation of back mutations. Back mutations may be caused by $\mathrm{AT} \rightarrow \mathrm{TA}$ transversion at the 3'ATT5' sequence in the TAA ochre codon but also by AT $\rightarrow$ GC transition at the last thymine of the same sequence. In the latter case, mRNA formed on this sequence contains the nonsense UAG amber codon recognized by supE $44_{\mathrm{am}}$ suppressor, normally present in AB1157 strain.

Phenotypically, growth-dependent $\mathrm{Arg}^{+}$ revertants of the $\mathrm{AB} 1157$ mut $T$ strain belonged mainly to class II but both classes, I and II, consisted of back mutations at the $\operatorname{argE3}$ locus. Adaptive $\mathrm{Arg}^{+}$revertants of this strain were back mutations too but, in opposition to the growth-dependent revertants, they belonged mainly to class I and within this

Table 1. Nucleotide sequence change associated with the $\mathrm{Arg}^{+}$phenotype of revertants of AB1157argE3 strain

\begin{tabular}{|c|c|c|c|c|c|c|}
\hline \multirow{2}{*}{ Site of mutation (suppressor) } & \multicolumn{5}{|c|}{ Lysis of $\mathrm{Arg}^{+}$revertants by T4 phages * } & \multirow{2}{*}{ Mutation } \\
\hline & WT & B17 & oc427 & ps292 & ps205 & \\
\hline $\sup B$ & + & + & + & + & + & $\mathrm{GC} \rightarrow \mathrm{AT}$ \\
\hline $\sup E_{(\mathrm{oc})}$ & + & - & + & + & + & $\mathrm{GC} \rightarrow \mathrm{AT}$ \\
\hline $\operatorname{argE3}$ & + & + & - & - & - & $* *$ \\
\hline supL & + & + & + & + & - & $\mathrm{AT} \rightarrow \mathrm{TA}$ \\
\hline
\end{tabular}

*Lysis (+) or nonlysis (-) was determined by placing $1-1.5 \times 10^{4}$ PFU of phage suspension on bacterial stripe on LB plate and incubating overnight. The T4 phages were: wild type (WT), amber (B17) or ochre (oc427, ps292, ps205).

${ }^{* *}$ Mutations in $\operatorname{argE} 3$ locus (back mutations) could be caused by transversions or transitions at the AT base pairs in the TAA ochre codon.

sitive to the set of T4 ochre mutants arose by back mutation in $\operatorname{argE3}$ gene.

Table 2 summarizes the results of specificity tests for growth-dependent and starvation-associated $\mathrm{Arg}^{+}$revertants of the AB1157 wild type, mutY and mutT strains. In the class a small fraction (around 12\%) of GC $\rightarrow \mathrm{AT}$ transitions was also observed.

In the AB1157mutY strain the level of growth-dependent $\mathrm{Arg}^{+}$revertants was low and phenotypic distribution of these two classes was similar to that characteristic for 
Table 2. Specificity of growth-dependent and adaptive $\mathrm{Arg}^{+}$revertants of the AB1157 strain

\begin{tabular}{|c|c|c|c|c|c|c|c|c|c|}
\hline \multirow[b]{3}{*}{ Strain } & \multirow{3}{*}{$\begin{array}{l}\text { Pheno- } \\
\text { typic } \\
\text { class }\end{array}$} & \multicolumn{8}{|c|}{ Type of mutations } \\
\hline & & \multicolumn{4}{|c|}{$\mathrm{Arg}^{+}$growth-dependent revertants per $10^{8}$ cells } & \multicolumn{4}{|c|}{$\begin{array}{l}\mathrm{Arg}^{+} \text {starvation-associated revertants } \\
\text { per } 10^{8} \text { cells }\end{array}$} \\
\hline & & $\begin{array}{l}\text { Distribution } \\
\text { among } \\
\text { phenotypic } \\
\text { classes (\%) }\end{array}$ & back & $\begin{array}{l}\sup B \text { by } \\
\mathrm{GC} \rightarrow \mathrm{AT} \\
\text { or sup } E_{\text {oc }}\end{array}$ & $\begin{array}{l}\text { supL by } \\
\mathrm{AT} \rightarrow \mathrm{TA}\end{array}$ & $\begin{array}{l}\text { Distribution } \\
\text { among } \\
\text { phenotypic } \\
\text { classes (\%) }\end{array}$ & back & $\begin{array}{l}\text { supB by } \\
\mathrm{GC} \rightarrow \mathrm{AT} \\
\text { or } \\
\text { supEoc }\end{array}$ & $\begin{array}{l}\text { supL by } \\
\mathrm{AT} \rightarrow \mathrm{TA}\end{array}$ \\
\hline \multirow[t]{2}{*}{ AB1157 } & I & 44 & - & 1.0 & 0.26 & 84 & 11 & 1.3 & - \\
\hline & II & 56 & - & 0.17 & 1.5 & 16 & 2.2 & - & 0.24 \\
\hline \multirow[t]{2}{*}{$\begin{array}{l}\text { AB1157 } \\
\text { mutT }\end{array}$} & I & 28 & 157 & - & - & 64 & 870 & 218 & - \\
\hline & II & 72 & 403 & -- & - & 36 & 612 & - & - \\
\hline \multirow[t]{2}{*}{$\begin{array}{l}\text { AB1157 } \\
\text { mutY }\end{array}$} & I & 27 & 1.5 & 0.38 & - & 99 & 79.2 & 63.4 & 15.8 \\
\hline & II & 73 & 2.6 & 1.0 & 1.5 & 1 & 1.6 & - & - \\
\hline
\end{tabular}

the mutT strain (class I, 27\%; class II, 73\%). Class I of the revertants of AB1157mutY strain consisted mainly (80\%) of back mutations and a much lower level (20\%) of GC $\rightarrow$ AT transitions. Class II consisted of back mutations (50\%) and equal amounts of $\mathrm{GC} \rightarrow \mathrm{AT}$ transitions and $\mathrm{AT} \rightarrow \mathrm{TA}$ transversions $(25 \%$ each). However, almost all (99\%) of the starvation- associated $\mathrm{Arg}^{+}$revertants of the $\mathrm{mut} Y$ strain belonged to class I and half of them were back mutations. The other half consisted of $\mathrm{GC} \rightarrow \mathrm{AT}$ transitions $(80 \%)$ and $\mathrm{AT} \rightarrow \mathrm{TA}$ transversions (20\%).

The results presented here are in agreement with the observation that adaptive mutations occur in the gene whose function was selected: phenotypically, colonies growing on E-Arg plates were $\mathrm{Arg}^{+}$but still $\mathrm{His}^{-}$and $\mathrm{Thr}^{-}$.

The adaptive class I $\mathrm{Arg}^{+}$reversions of the mut $T$ strain consisted of back mutations ( $80 \%)$ and mutations which arose as a result of $\sup B$ suppressor formation by $\mathrm{GC} \rightarrow \mathrm{AT}$ transitions (20\%). All of the adaptive $\mathrm{Arg}^{+}$revertants of the mut $Y$ strain belonged to class I; half of them consisted of back mutations and the other half resulted from supB (40\%) and supL (10\%) suppressors formation by GC $\rightarrow$ AT transitions and $\mathrm{AT} \rightarrow \mathrm{TA}$ transversions, respectively. This means that the adaptive and growth-dependent reversions of arginine auxotrophy in the mutY strain arose by the formation of different suppressors.

\section{CONCLUSIONS}

The presented here $\arg E 3 \rightarrow \mathrm{Arg}^{+}$reversion to prototrophy is a valuable system for investigation of adaptive mutations. An additional advantage of the system is that, using a set of T4 phage mutants and two additional markers: hisG4 and thr-1, it is possible to estimate the specificity of the mutations (type of suppressor leading to $\mathrm{Arg}^{+}$mutation) and distinguish between $\mathrm{Arg}^{+}$growth-dependent and adaptive revertants. This was demonstrated for the AB1157 mutY strain. The AB1157 mut $T$ we used here as a tester strain, it did not show the advantage of the system because almost all of its $\mathrm{Arg}^{+}$revertants resulted from back mutations in the argE3 gene. Nevertheless, there are strains (for instance AB1157 mutated in $d n a Q$ gene encoding the $\varepsilon$ subunit of DNA polymerase III, Grzesiuk \& Nowosielska, in preparation), which revert to prototrophy by forming suppressors and in those strains the specificity of mutations can be precisely estimated. 
The application of replica plating for adaptive mutation investigation is a new and very useful idea. It shows directly the difference between growth-dependent (even small, slow growing) colonies and those which arose due to an adaptive mutation.

The authors wish to thank Drs I. Fijałkowska and W. Zagórski for their helpful comments and stimulating discussion.

\section{R E F E R E N C E S}

1. Cairns, J. \& Foster, P.L. (1991) Adaptive reversion of a frameshift mutation in Escherichia coli. Genetics 128, 695-701.

2. Cairns, J., Overbaugh, J. \& Miller, S. (1988) The origin of mutants. Nature 335, 142-145.

3. Foster, P.L. (1993) Adaptive mutation: The uses of adversity. Annu. Rev. Microbiol. 47, 467-504.

4. Hall, B.G. (1990) Spontaneous point mutations that occur more often when they are advantageous than they are neutral. Genetics $\mathbf{9 0}$, 673-691.

5. Ryan, F.J. (1955) Spontaneous mutations in non-dividing bacteria. Genetics 40, 726-310.

6. Foster, P.L. (1997) Nonadaptive mutations occur on the F' episome during adaptive mutation conditions in Escherichia coli. J. Bacteriol. 179, 1550-1554.

7. Foster, P.L. \& Trimarchi, J.M. (1995) Adaptive reversion of an episomal frameshift mutation in Escherichia coli requires conjugal functions but not actual conjugation. Proc. Natl. Acad. Sci. U.S.A. 92, 5487-5490.

8. Rosenberg, S.M., Longerich, S., Gee, G. \& Harris, R.S. (1994) Adaptive mutation by deletion in small mononucleotide repeats. Science $\mathbf{2 6 5}$, 405-407.

9. Foster, P.L. \& Trimarchi, J.M. (1994) Adaptive reversion of a frameshift mutation in
Escherichia coli by simple base substitutions in homopolymeric runs. Science 265, 407-409.

10. Bridges, B.A. (1993) Spontaneous mutation in stationary-phase Escherichia coli WP2 carrying various DNA repair alleles. Mutation Res. 302, 173-176.

11. Au, K.G., Clarke, G.S., Miller, J.H. \& Modrich, P. (1989) Escherichia coli mutY gene encodes an adenine glycosylase active on G-A mispairs. Proc. Natl. Acad. Sci. U.S.A. 86, 8877-8881.

12. Bridges, B.A. (1996) Elevated mutation rate in mutT bacteria during starvation: evidence for DNA turnover? J. Bacteriol. 178, 2709-2711.

13. Miller, J.H. (1972) Experiments in Molecular Genetics, pp. 56-59, Cold Spring Harbor Laboratory, Cold Spring Harbor, New York.

14. Bachmann, B.J. (1987) Derivations and genotypes of some mutant derivatives of Escherichia coli K-12; in Escherichia coli and Salmonella typhimurium: Cellular and Molecular Biology (Neidhard, F.C., Ingraham, J., Low, K.B., Magasanik, B., Schaechler, M. \& Umbarger, H.E., eds.) vol. 2, pp. 1190-1219, American Society for Microbiology, Washington D.C.

15. Wójcik, A., Grzesiuk, E., Tudek, B. \& Janion, C. (1996) Conformation of plasmid DNA from Escherichia coli deficient in the repair systems protecting DNA from 8-oxoguanine lesions. Biochimie 78, 85-89.

16. Vogel, H.J. \& Bonner, D.M. (1956) Acetyloornithinase of Escherichia coli: Partial purification and some properties. J. Biol. Chem. 218, 97-106.

17. Sargentini, N.J. \& Smith, K.C. (1989) Mutational spectrum analysis of $u m u C$-dependent $\gamma$-radiation mutagenesis in Escherichia coli. Mutation Res. 211, 93-203.

18. Śledziewska-Gójska, E., Grzesiuk, E., Płachta, A. \& Janion, C. (1992) Mutagenesis of Escherichia coli: A method for determining mutagenic specificity by analysis of tRNA suppressors. Mutagenesis 7, 41-46. 
19. Bridges, B.A. \& Ereira, S. (1998) DNA synthesis and viability of a $m u t T$ derivative of Escherichia coli WP2 under conditions of amino acid starvation and relation to stationary phase (adaptive) mutation. J. Bacteriol. 180, 2906-2910.

20. Maki, H. \& Sekiguchi, M. (1992) MutT protein specifically hydrolyses a potent mutagenic substrate for DNA synthesis. Nature 355, 273-275.

21. Nghiem, Y., Cabrera, M., Cupples, C.G. \& Miller, J.H. (1998) The mutY gene: A mutator locus in Escherichia coli that generates G:C $\rightarrow$ T:A transversions. Proc. Natl. Acad. Sci. U.S.A. 85, 2709-2713. 